\title{
论 文 化学热力学研究进展专刊
}

\section{铋系含氧酸盐改性镁水解制氢的动/热力学研究}

\author{
林杰 $^{\dagger}$, 刘佳溪 ${ }^{\dagger}$, 孙立贤* ${ }^{*}$ 徐芬 ${ }^{*}$, 罗玉梅, 夏永鹏, 张晨晨, 程日光, 魏胜, 黄鹏儒, \\ 李涁, 张可翔, 蔡丹
}

广西电子信息材料构效关系重点实验室, 广西新能源材料结构与性能协同创新中心, 桂林电子科技大学材料科学与工程学院, 桂林 541004 †同等贡献

*通讯作者, E-mail: sunlx@guet.edu.cn; xufen@guet.edu.cn

收稿日期: 2021-12-16; 接受日期: 2022-02-09; 网络版发表日期: 2022-04-12

国家重点基础研究发展计划(编号：2018YFB1502103，2018YFB1502105)、国家自然科学基金(编号：U20A20237，51863005，51462006, $51102230 ， 52101245 ， 51871065 ， 51971068$ )、广西八桂学者基金、中德国际合作项目(编号：GZ1528)、广西创新驱动发展专项(编号: AA19182014, AD17195073, AA17202030-1)和广西信息材料重点实验室(编号: 201009-Z, 211017-K, 211014-K, 211016-K, 211022-K, 211023K, 201016K)资助项目

摘要 $\mathrm{Mg}$ 基制氢材料具有来源广泛、反应温和、工艺简单、安全可控、理论产氢量高等优势, 是当今的研究 热点. 本文提出采用高能球磨方法制备 $\mathrm{Mg}$ - $\mathrm{Bi}$ 系含氧酸盐 $\mathrm{Bi}_{x} \mathrm{M}_{y} \mathrm{O}_{z}(\mathrm{M}=\mathrm{Ti}, \mathrm{V}, \mathrm{Cr}, \mathrm{Mo}, \mathrm{W})$ 复合材料以改善 $\mathrm{Mg}$ 水解 制氢性能. 本工作研究发现, 掺杂 $\mathrm{Bi}_{2} \mathrm{MoO}_{6}$ 的 $\mathrm{Mg}$ 基复合制氢材料具有较好的性能, $\mathrm{Mg}-7 \mathrm{wt} \% \mathrm{Bi}_{2} \mathrm{MoO}_{6}$ 在 $298.15 \mathrm{~K}$ 的最大产氢速率为 $756.1 \mathrm{~mL} \mathrm{~g}^{-1} \mathrm{~min}^{-1}$. 通过引入多壁碳纳米管 $(\mathrm{CNTs})$ 可以进一步改善 $\mathrm{Mg}-\mathrm{Bi}_{2} \mathrm{MoO}_{6}$ 的产氢性能, $\mathrm{Mg}-7 \mathrm{wt} \% \mathrm{Bi}_{2} \mathrm{MoO}_{6} / \mathrm{CNTs}$ 的最大产氢速率达 $2172.4 \mathrm{~mL} \mathrm{~g}^{-1} \mathrm{~min}^{-1}$, 产氢活化能下降至 $23.6 \mathrm{~kJ} \mathrm{~mol}^{-1}$. X光电子能谱 (XPS)分析表明 $\mathrm{Bi}_{2} \mathrm{MoO}_{6} / \mathrm{CNTs}$ 与 $\mathrm{Mg}$ 在球磨过程中发生固相反应生成 $\mathrm{Bi}$ 单质. 密度泛函理论(DFT)计算揭示 $\mathrm{Bi}$ 原子 掺杂可改变 $\mathrm{Mg}$ 的局域电荷分布, 增强 $\mathrm{Mg}$ 对 $\mathrm{H}_{2} \mathrm{O}$ 的吸附能, 并降低 $\mathrm{H}_{2} \mathrm{O}$ 解离后 $\mathrm{H}$ 原子的吸附能, 促进水解反应进行.

关键词水解制氢, $\mathrm{Mg}$, 铋系含氧酸盐, 动力学, 热力学

\section{1 引言}

随着社会不断发展，人们对能源的需求日益增加. 目前世界上的能源主要来自于化石能源, 如煤炭、石油 和天然气等不可再生资源. 化石能源的使用导致大气中 的二氧化碳含量急剧上升, 造成了严峻的环境问题 ${ }^{[1,2]}$.

相比于化石能源，氢气作为一种高效、绿色、可 再生的二次能源具有来源广泛、环境友好、燃烧热值 高(完全燃烧的热值达 $141.8 \mathrm{MJ} \mathrm{Kg}^{-1}$ )、易于和其他能 源转换等优点 ${ }^{[3 \sim}$. 因此, 氢气可作为传统化石能源过
渡到大规模应用可再生能源之间的理想中间媒介，有 着良好的发展前景.

目前，氢气的制取途径主要为化石燃料裂解 ${ }^{[6]}$ 、 电解水 ${ }^{[7]}$ 和生物制氢 ${ }^{[8,9]}$ 等, 这些方法存在转换效率 低、能耗大、成本高等缺点. 因此，传统的制-储氢技 术不能满足绿色“氢经济”的要求. 金属氢化物的固态 储氢技术具有安全性高、储氢密度大和可逆性好等优 势 ${ }^{[10,11]}$, 但其热解放氢所需的温度与压强等条件仍较 为苛刻. 利用活泼金属与水快速反应制备氢气的方法, 具有携带方便、存储容易等优点，成为近年来制氢领

引用格式: Lin J, Liu J, Sun L, Xu F, Luo Y, Xia Y, Zhang C, Cheng R, Wei S, Huang P, Li B, Zhang K, Cai D. Kinetic/thermodynamic study on improved hydrolysis hydrogen generation of Mg-doped bismuth series oxysalt. Sci Sin Chim, 2022, 52: 689-700, doi: 10.1360/SSC-2021-0251 
域的研究热点 ${ }^{[12]}$.

$\mathrm{Li} 、 \mathrm{Na} 、 \mathrm{~K}$ 等碱金属还原性强，与水反应可快速 析出氢气，但其反应过于剧烈而难以控制，并具有安 全隐患. $\mathrm{Al} 、 \mathrm{Mg}$ 的水解放氢反应条件温和, 产氢量高, 备受研究者的青睐 ${ }^{[12 ~ 15]} . \mathrm{Mg}$ 与水反应的理论产氢量可 达 $933 \mathrm{~mL} \mathrm{~g}^{-1}$. 但在其水解反应过程中, 生成难溶的 $\mathrm{Mg}(\mathrm{OH})_{2}$ 会迅速覆盖在 $\mathrm{Mg}$ 的表面，阻碍 $\mathrm{Mg}$ 与水持续 反应, 从而影响氢气生成的速度和转换率 ${ }^{[16]}$.

改善 $\mathrm{Mg}$ 的水解制氢性能，有以下方法: 通过球磨 加入过渡金属类掺杂剂 $[17,18]$ 、将 $\mathrm{Mg}$ 与低熔点金属或 其他金属形成合金 ${ }^{[19-21]}$ 、改变反应溶液 ${ }^{[22-24]}$ 等. 其中, 通过球磨掺杂添加剂的方法工艺简便, 可以增加 $\mathrm{Mg}$ 的 缺陷和新的表面 ${ }^{[17]}$, 从而提高水解反应的动力学. 此 外，引入掺杂剂可以改变 $\mathrm{Mg}$ 在溶液中的反应环境，增 加水解反应的化学活性位点等 ${ }^{[25]}$. 文献报道的 $\mathrm{Mg}$ 水解 制氢材料的球磨掺杂剂主要有 $\mathrm{AlCl}_{3} 、 \mathrm{CoCl}_{2}$ $\mathrm{CuCl}^{[26-28]}$ 等卤化物, 此类掺杂剂可以有效减小材料尺 寸并且改变反应溶液环境, $\mathrm{Nb}_{2} \mathrm{O}_{5} 、 \mathrm{~V}_{2} \mathrm{O}_{5} 、 \mathrm{MoO}_{3}{ }^{[17,29]}$ 等氧化物可使材料在球磨后表面产生更多缺陷与新的 表面，石墨、膨胀石墨、碳纤维等碳材料 ${ }^{[17,30]}$ 则具有 较高的化学稳定性与优良的电催化活性可以促进 $\mathrm{Mg}$ 水解反应, 以及 $\mathrm{NaBH}_{4}$ 和 $\mathrm{MoS}_{2}$ 等 ${ }^{[25,29]}$. 但是, 上述催化 剂不能同时兼顾水解反应动力学和体系转化率, 仍需 进一步探究. 根据报道, $\mathrm{Bi}$ 系化合物能够有效改善 $\mathrm{Al}$ 基材料水解反应性能 ${ }^{[31]}$, 而对 $\mathrm{Mg}$ 基材料水解反应的 研究未见报道.

本文采用球磨制备 $\mathrm{Mg}-\mathrm{Bi}$ 系含氧酸盐 $\mathrm{Bi}_{x} \mathrm{M}_{y} \mathrm{O}_{z}$ $(\mathrm{M}=\mathrm{Ti}, \mathrm{V}, \mathrm{Cr}, \mathrm{Mo}, \mathrm{W})$ 复合材料以改善 $\mathrm{Mg}$ 水解制氢性 能. 通过水热法制备了 $\mathrm{Bi}_{2} \mathrm{MoO}_{6} /$ 多壁碳纳米管 $(\mathrm{CNTs})$ 复合掺杂剂, 并采用机械球磨将其添加到 $\mathrm{Mg}$ 中, 以进 一步改善 $\mathrm{Mg}$ 水解反应制氢性能，降低 $\mathrm{Mg}$ 制氢材料水 解反应的活化能. 本工作研究了复合材料水解制氢的 热力学和动力学性能, 通过X光电子能谱(XPS)、扫描 电子显微镜(SEM)等分析仪器表征了复合材料的化学 组成和微观结构, 并结合密度泛函理论(DFT)计算揭示 了 $\mathrm{Bi}_{2} \mathrm{MoO}_{6}$ 对 $\mathrm{Mg}$ 的催化水解制氢机理.

\section{2 实验部分}

\section{1 试剂与材料}

钛酸四丁酯 $\left(\mathrm{Ti}\left(\mathrm{C}_{4} \mathrm{H}_{9} \mathrm{O}\right)_{4}\right)$ 为化学纯, 柠檬酸铋铵
$\left(\mathrm{C}_{6} \mathrm{H}_{10} \mathrm{BiNO}_{8}\right)$ 为分析纯, 氢氧化钠 $(\mathrm{NaOH})$ 为分析纯, 铬酸钠 $\left(\mathrm{Na}_{2} \mathrm{CrO}_{4}\right)$ 为化学纯, 铇酸钠二水合物 $\left(\mathrm{Na}_{2} \mathrm{WO}_{4}\right)$ 为分析纯, 购自阿拉丁试剂(上海)有限公司(中国); 十 二烷基苯磺酸钠(SDBS) 为化学纯, 偏钒酸铵 $\left(\mathrm{NH}_{4} \mathrm{VO}_{3}\right)$ 为分析纯, 硝酸 $\left(\mathrm{HNO}_{3}\right)$ 质量分数为 $65.0 \% \sim 68.0 \%$, 购自 西陇化工股份有限公司(中国); 无水乙醇、钼酸钠二 水合物 $\left(\mathrm{Na}_{2} \mathrm{MoO}_{4}\right)$ 为分析纯, 购自广东光华科技股份 有限公司 (中国); 五水合硝酸铋 $\left(\mathrm{Bi}\left(\mathrm{NO}_{3}\right)_{3} \cdot 5 \mathrm{H}_{2} \mathrm{O}\right)$ 为分 析纯, 氯化钠 $(\mathrm{NaCl})$ 为分析纯, 多壁碳纳米管 $(\mathrm{CNTs})$, 购自北京伊诺凯科技有限公司(中国).

\section{2 实验方法}

(1) $\mathrm{Bi}$ 系含氧酸盐的制备. 将 $\mathrm{Ti}\left(\mathrm{C}_{4} \mathrm{H}_{9} \mathrm{O}\right)_{4}$ 加入到 $30 \mathrm{~mL}$ 的 $3 \mathrm{~mol} \mathrm{~L}^{-1} \mathrm{NaOH}$ 溶液中超声搅拌, 然后加入 摩尔比为 $\mathrm{Bi}: \mathrm{Ti}=4: 3$ 的 $\mathrm{Bi}\left(\mathrm{NO}_{3}\right)_{3} \cdot 5 \mathrm{H}_{2} \mathrm{O}$, 连续超声摚拌 $30 \mathrm{~min}$ 后转移到高温反应釜的聚四氟乙烯衬里密封, 在 $180^{\circ} \mathrm{C}$ 下保持 $20 \mathrm{~h}$, 最后经过洗涤过滤获得 $\mathrm{Bi}_{4} \mathrm{Ti}_{3} \mathrm{O}_{12}$; 将 $1 \mathrm{mmol} \mathrm{Bi}\left(\mathrm{NO}_{3}\right)_{3} \cdot 5 \mathrm{H}_{2} \mathrm{O}, 1 \mathrm{mmol} \mathrm{NH}_{4} \mathrm{VO}_{3}$ 和 $0.1 \mathrm{~g}$ 的 SDBS 分别溶解在 $6 \mathrm{~mL}, 6 \mathrm{~mL}$ 和 $5 \mathrm{~mL}$ 的 $2 \mathrm{~mol} \mathrm{~L}^{-1}$ $\mathrm{HNO}_{3}$ 溶液中, 然后将三溶液混合剧烈搅拌得到混合 溶液, 再将混合溶液转移至高温反应釜的聚四氟乙烯 祄里密封, 在 $150^{\circ} \mathrm{C}$ 下保持 $12 \mathrm{~h}$, 最后经过洗涤过滤获 得 $\mathrm{BiVO}_{4}$; 将 $4 \mathrm{mmol} \mathrm{Bi}\left(\mathrm{NO}_{3}\right)_{3} \cdot 5 \mathrm{H}_{2} \mathrm{O}$ 和 $2 \mathrm{mmol} \mathrm{Na}_{2} \mathrm{MO}_{4}$ $(\mathrm{M}=\mathrm{W}, \mathrm{Mo}$ 或 $\mathrm{Cr})$ 溶解在 $80 \mathrm{~mL}$ 去离子水中搅拌 $30 \mathrm{~min}$ 后转移到高温反应釜的聚四氟乙烯祄里密封, 在 $180^{\circ} \mathrm{C}$ 下保持 $20 \mathrm{~h}$, 最后经过洗涤过滤获得 $\mathrm{Bi}_{2} \mathrm{CrO}_{6}$ 、 $\mathrm{Bi}_{2} \mathrm{MoO}_{6}$ 和 $\mathrm{Bi}_{2} \mathrm{WO}_{6}$; 在水热法制备 $\mathrm{Bi}_{2} \mathrm{MoO}_{6}$ 过程中加 入超声分散 $30 \mathrm{~min}$ 的 CNTs, 其中 $\mathrm{Bi}_{2} \mathrm{MoO}_{6}$ 与 $\mathrm{CNTs}$ 质量 比为6:1, 制备催化剂 $\mathrm{Bi}_{2} \mathrm{MoO}_{6} / \mathrm{CNTs}$ 复合催化剂.

(2) $\mathrm{Mg}$ 基粉体制氢材料的制备. 在氩气环境保护 下, 将 $\mathrm{Mg}$ 粉和 $\mathrm{Bi}$ 系含氧酸盐按照 $9: 1$ 质量比投入到球 磨罐中, 其中球料比为 $20: 1$, 转速为 $250 \mathrm{r} \mathrm{min}^{-1}$, 球磨 时间为30 120 min, 得到均匀混合的 $\mathrm{Mg}$ 基制氢材料.

(3) X射线衍射(XRD)分析. 利用X射线衍射仪对 $\mathrm{Bi}$ 系含氧酸盐的结构分析. 仪器型号为德国布鲁克公司 的D8-Advance. 实验参数为: Cu靶, 工作电压为 $40 \mathrm{kV}$, 工作电流为 $40 \mathrm{~mA}$, 扫描范围为 $10^{\circ} \sim 90^{\circ}$, 扫描速度为 $10^{\circ} \mathrm{min}^{-1}$, 步长为 $0.01^{\circ} \sim 0.02^{\circ}$.

(4) SEM表征. 采用场发射扫描电子显微镜来对材 料的微观形貌进行分析. 实验所用的仪器为美国FEI公 司的Quanta FEG 450; 实验是在真空环境下, 高速交流

690 
电压为5 10 kV进行.

(5) TEM表征. 采用TEM对Bi系含氧酸盐的显微结 构进行观测及微区元素进行分析. 所用仪器为美国FEI 公司的 Talos F200X透射电子显微镜, 操作电压为 $200 \mathrm{kV}$, 点分辨率为 $0.25 \mathrm{~nm}$, 信息分别率为 $0.12 \mathrm{~nm}$.

(6) XPS表征. 采用美国赛默飞Escalab 250Xi型X 射线光电子能谱对样品进行定性和半定量分析, 通过 XPS图谱分析得到材料表面元素的种类、价态和 含量.

(7) 反应热测量. 采用法国塞塔拉姆公司的 $\mathrm{C} 80$ 量 热仪对材料水解反应热进行测量, 选用的样品池为破 膜混合池, 实验温度为 $298.15 \mathrm{~K}$, 在混合池内装入约 $0.01 \mathrm{~g}$ 制氢材料, 通过对热流曲线基线积分求出水解 反应热。

(8) 制氢材料产氢性能测试. 测量氢气产生的装置 与本课题组之前工作 ${ }^{[31]}$ 相同, 原理为排水集气法. 根 据测量排出水的质量, 可得产生的气体的体积, 即为 材料与水反应放出氢气的体积. 具体操作为每次在手 套箱中称取约 $0.1 \mathrm{~g}$ 的待测样品装入反应池中, 将装好 样品的反应池置于恒温水浴锅中, 温度恒定后向反应 池中注入 $10 \mathrm{~mL}$ 具有同等温度的溶液; 产生的气体经 过冷凝, 干燥后进入集气瓶中; 采用电子天平记录水 的质量变化量, 设定电子天平 $1 \mathrm{~s}$ 读取一个数据, 并通 过电脑自动记录排出的水的质量. 为了避免实验误差, 在测试之前进行空白实验, 测出的放氢量均扣除了空 白值. 此外, 每个样品重复测试至少 3 次以上, 确保误 差值在 $\pm 5 \%$ 之内.

(9) DFT计算. 基于DFT框架下的维也纳从头算软 件包VASP进行模拟计算. 采用投影缀加波(PAW)来考

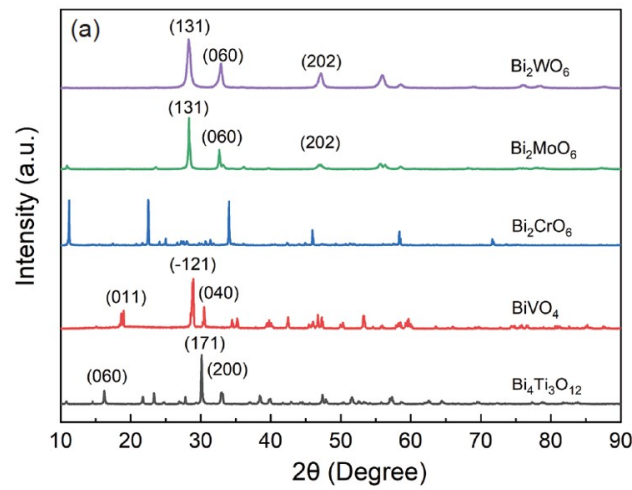

虑电子-离子相互作用, 广义梯度近似(GGA)中的PBE 泛函描述电子的交换关联势.

\section{3 结果与讨论}

\subsection{Mg-Bi系含氧酸盐 $\mathrm{Bi}_{x} \mathrm{M}_{y} \mathrm{O}_{z}$ 的结构与产氢性能 分析}

对水热法制备的铋系含氧酸盐进行XRD表征，如 图1a所示, 水热法合成出相应的铋系含氧酸盐具有与 标准材料相同的特征峰, 且峰较为尖锐, 表明材料成 功制备, 具有较高的结晶性. Mg-铋系含氧酸盐的XRD 表征如图 $1 \mathrm{~b}$ 所示, 各制氢材料不仅显示出 $\mathrm{Mg}$ 的特征 峰, 还存在着各铋系含氧酸盐的特征峰且各特征峰没 有发生明显的变化, 表明在球磨过程中大部分铋系含 氧酸盐与 $\mathrm{Mg}$ 没有发生反应, 从 $\mathrm{Mg}-10 \mathrm{wt} \% \mathrm{Bi}_{2} \mathrm{MoO}_{6}$ 与 Mg-10 wt \% Bi ${ }_{2} \mathrm{MoO}_{6} / \mathrm{CNTs}$ 的XRD图对比可以发现加 入 CNTs样品的 $\mathrm{Bi}_{2} \mathrm{MoO}_{6}$ 特征峰更明显, 说明加入 CNTs 可使样品中的 $\mathrm{Bi}_{2} \mathrm{MoO}_{6}$ 保持较高的结晶度. 使用自制 的产氢性能评价装置, 在 $298.15 \mathrm{~K}$ 反应温度下对制氢 材料进行产氢性能评价, 各Bi系含氧酸盐与 $\mathrm{Mg}$ 材料产 氢性能如表1所示. 向反应容器注入 $10 \mathrm{~mL} 3.5 \mathrm{wt} \%$ $\mathrm{NaCl}$ 溶液后, 氢气开始释放并进行计数. 其中氢气产 生的诱导阶段时间受环境温度、添加剂类型和粉末尺 寸的强烈影响. 产氢曲线如图2所示, 结合表1, 可以看 出各 $\mathrm{Bi}$ 系含氧酸盐与 $\mathrm{Mg}$ 材料均有较好的产氢性能, 其 中 $10 \% \mathrm{Bi}_{2} \mathrm{MoO}_{6}$ 的催化效果最好, 最大产氢速率可达 到 $564 \mathrm{~mL} \mathrm{~g}^{-1} \mathrm{~min}^{-1}$, 产氢量为 $830.5 \mathrm{~mL} \mathrm{~g}^{-1}$, 产氢率为 $98.9 \%$, 这表明 $\mathrm{Mg}$ 粉末通过加入 $\mathrm{Bi}$ 系含氧酸盐都有较 高的氢气转化率, 其中加入 $\mathrm{Bi}_{2} \mathrm{MoO}_{6}$ 球磨可以更有效

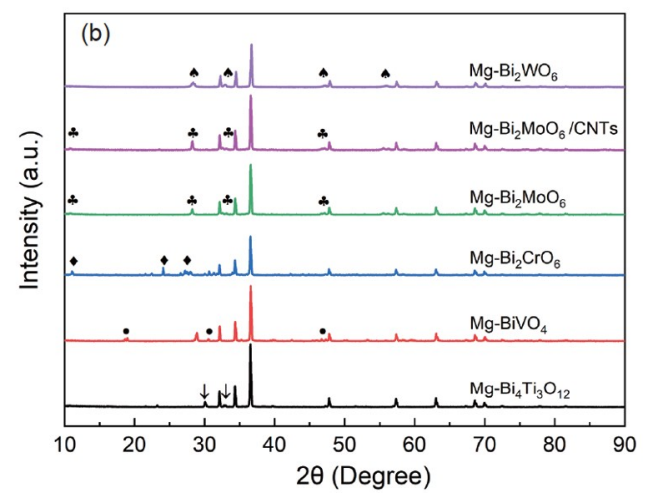

图 1 XRD图谱. (a) Bi系含氧酸盐; (b) Mg-Bi系含氧酸盐(网络版彩图)

Figure 1 XRD pattern of (a) bismuth series oxysalt and (b) Mg-bismuth series oxysalt (color online). 
表 1 各种 $10 \mathrm{wt} \% \mathrm{Bi}$ 系含氧酸盐球磨 $\mathrm{Mg}$ 产氢性能

Table 1 The hydrogen generation performances of ball milled Mg-10 $\mathrm{wt} \% \mathrm{Bi}_{x} \mathrm{M}_{y} \mathrm{O}_{z}$ at $298.15 \mathrm{~K}$

\begin{tabular}{cccc}
\hline 样品 & $\begin{array}{c}\text { 最大产氢速率 } \\
\left(\mathrm{mL} \mathrm{g}^{-1} \mathrm{~min}^{-1}\right)\end{array}$ & $\begin{array}{c}\text { 产氢量 } \\
\left(\mathrm{mL} \mathrm{g}^{-1}\right)\end{array}$ & $\begin{array}{c}\text { 产氢率 } \\
(\%)\end{array}$ \\
\hline $\mathrm{Mg}$ & 62 & 252.9 & 27.1 \\
$\mathrm{Mg}-10 \mathrm{wt} \% \mathrm{Bi}_{4} \mathrm{Ti}_{3} \mathrm{O}_{12}$ & 396.2 & 812.1 & 96.7 \\
$\mathrm{Mg}-10 \mathrm{wt} \% \mathrm{BiVO}_{4}$ & 300.7 & 779.5 & 92.8 \\
$\mathrm{Mg}-10 \mathrm{wt} \% \mathrm{Bi}_{2} \mathrm{CrO}_{6}$ & 162.5 & 759.2 & 90.4 \\
$\mathrm{Mg}-10 \mathrm{wt} \% \mathrm{Bi}_{2} \mathrm{MoO}_{6}$ & 564 & 830.5 & 98.9 \\
$\mathrm{Mg}-10 \mathrm{wt} \% \mathrm{Bi}_{2} \mathrm{WO}_{6}$ & 112.1 & 751.7 & 89.5 \\
\hline
\end{tabular}

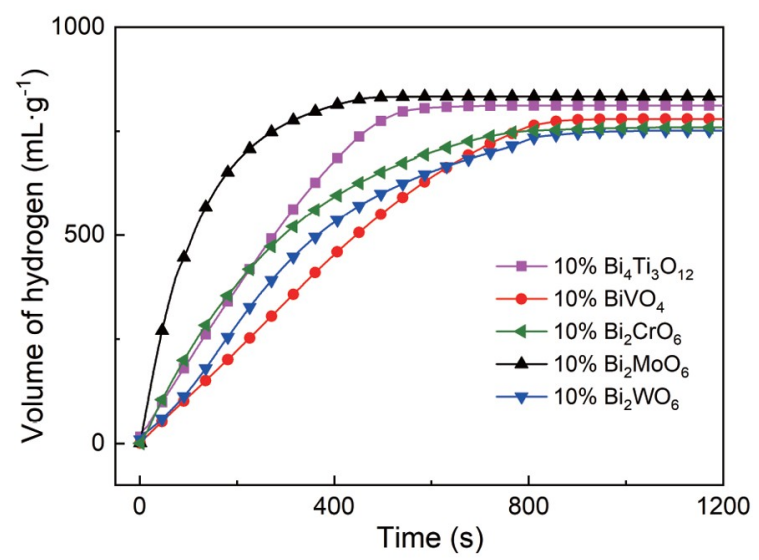

图 $2 \mathrm{Mg}-\mathrm{Bi}_{x} \mathrm{M}_{y} \mathrm{O}_{z}$ 的产氢性能(网络版彩图)

Figure 2 The amount of hydrogen generation for bismuth series oxysalt (color online).

地活化制氢材料进行水解反应.

\section{$3.2 \mathrm{Mg}-\mathrm{Bi}$ 系含氧酸盐 $\mathrm{Bi}_{x} \mathrm{M}_{y} \mathrm{O}_{z}$ 的产氢动力学分析}

温度对 $\mathrm{Mg}$ 的水解反应具有较大影响, 为了进一步 研究反应温度对水解的影响, 将球磨 $60 \mathrm{~min}$ 的 Mg-10 $\mathrm{wt} \% \mathrm{Bi}_{4} \mathrm{Ti}_{3} \mathrm{O}_{12} 、 \mathrm{Mg}-10 \quad \mathrm{wt} \% \mathrm{BiVO}_{4} 、 \mathrm{Mg}-10 \quad \mathrm{wt} \%$ $\mathrm{Bi}_{2} \mathrm{CrO}_{6} 、 \mathrm{Mg}-10 \mathrm{wt} \% \mathrm{Bi}_{2} \mathrm{MoO}_{6}$ 和 $\mathrm{Mg}-10 \mathrm{wt} \% \mathrm{Bi}_{2} \mathrm{WO}_{6}$ 分别在288.15、298.15、308.15、318.15和328.15 K的 $3.5 \mathrm{wt} \% \mathrm{NaCl}$ 溶液进行水解反应. 如表 2 所示, $\mathrm{Mg}$ 制氢 材料在 $1200 \mathrm{~s}$ 内进行水解反应, 随着水解反应温度升 高所有添加 $\mathrm{Bi}$ 系含氧酸盐的 $\mathrm{Mg}$ 基制氢材料的转化率 都相应增加. 值得注意的是, $\mathrm{Mg}-10 \mathrm{wt} \% \mathrm{Bi}_{2} \mathrm{MoO}_{6}$ 在反 应温度 $318.15 \mathrm{~K}$ 以上时氢气转化率可达到 $100 \%$, 表 3 为 制氢材料在288.15 318.15 K的最大产氢速率. 同样地, 随着水解反应温度升高所有添加 $\mathrm{Bi}$ 系含氧酸盐的 $\mathrm{Mg}$ 基制氢材料的最大产氢速率也都相应增加，说明提高 水解反应温度可以有效提高 $\mathrm{Mg}$ 水解产氢动力学性能.

$\mathrm{Mg}$ 基材料水解的产氢动力学性能可以通过阿伦 尼乌斯公式(Arrhenius equation)推算出来的活化能进 行评估. 公式如下:

$k=A \cdot \exp \left(-E_{\mathrm{a}} / \mathrm{R} T\right)$

其中 $k$ 是水解反应的最大产氢速率 $\left(\mathrm{mL} \mathrm{g}^{-1} \mathrm{~min}^{-1}\right), A$ 为 指数因子, $E_{\mathrm{a}}$ 是反应的活化能 $\left(\mathrm{kJ} \mathrm{mol}^{-1}\right), R$ 是 $8.314 \mathrm{~J} \mathrm{~mol}^{-1} \mathrm{~K}^{-1}, T$ 是绝对温度 $(\mathrm{K})$. 根据阿伦尼乌斯 公式, 我们绘制了不同的 $\mathrm{Bi}$ 系含氧酸盐的 $\mathrm{Mg}$ 制氢材料 的反应速率和对应的反应温度 $\ln k-1000 / T$ 曲线, 如图3 所示. 由图中直线的斜率可计算出各 $\mathrm{Mg}-10 \mathrm{wt} \% \mathrm{Bi}$ 系 含氧酸盐与水反应的表观活化能，其中 $\mathrm{Mg}-10 \mathrm{wt} \%$ $\mathrm{Bi}_{2} \mathrm{MoO}_{6}$ 在 $3.5 \mathrm{wt} \% \mathrm{NaCl}$ 溶液的表观活化能 $\left(36.8 \mathrm{~kJ} \mathrm{~mol}^{-1}\right)$ 远低于 $\mathrm{Mg}$ 在 $3.5 \mathrm{wt} \% \mathrm{NaCl}$ 溶液的表 观活化能 $\left(65.3 \mathrm{~kJ} \mathrm{~mol}^{-1}\right)$ 与 $\mathrm{Mg}$ 在海水中进行水解反

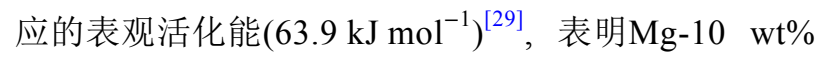
$\mathrm{Bi}_{2} \mathrm{MoO}_{6}$ 制氢材料具有较好的反应活性.

\section{3 $\mathrm{Bi}_{2} \mathrm{MoO}_{6}$ 含量对 $\mathrm{Mg}$ 基材料的产氢性能影响}

我们对 $\mathrm{Mg}-x \mathrm{wt} \% \mathrm{Bi}_{2} \mathrm{MoO}_{6}(x=3,5,7,10,13)$ 在

表 2 不同水解温度下 $10 \mathrm{wt} \% \mathrm{Bi}$ 系含氧酸盐球磨 $\mathrm{Mg}$ 转化率

Table 2 Conversion yield of hydrogen generation of Mg doped with bismuth series oxysalt by ball milling at different temperatures

\begin{tabular}{cccccc}
\hline \multirow{2}{*}{$\mathrm{Mg}-10 \mathrm{wt} \% \mathrm{Bi}_{x} \mathrm{M}_{y} \mathrm{O}_{z}$} & \multicolumn{5}{c}{ 转化率 (\%) } \\
\cline { 2 - 6 } & $288.15 \mathrm{~K}$ & $298.15 \mathrm{~K}$ & $308.15 \mathrm{~K}$ & $318.15 \mathrm{~K}$ & $328.15 \mathrm{~K}$ \\
\hline $\mathrm{Bi}_{4} \mathrm{Ti}_{3} \mathrm{O}_{12}$ & 91.5 & 96.7 & 97.8 & 98.5 & 99.5 \\
$\mathrm{BiVO}_{4}$ & 83.5 & 92.8 & 97.3 & 97.7 & 97.9 \\
$\mathrm{Bi}_{2} \mathrm{CrO}_{6}$ & 80.3 & 90.4 & 95.8 & 97.5 & 90 \\
$\mathrm{Bi}_{2} \mathrm{MoO}_{6}$ & 95.1 & 99.2 & 99.4 & 96.8 & 100 \\
$\mathrm{Bi}_{2} \mathrm{WO}_{6}$ & 80.8 & 89.5 & 96.9 & 97.8 & 98.2 \\
\hline
\end{tabular}


表 3 不同温度下掺杂 $\mathrm{Bi}$ 系含氧酸盐的 $\mathrm{Mg}$ 最大产氢速率

Table 3 Hydrogen generation rate of $\mathrm{Mg}$ doped with bismuth series oxysalt by ball milling at different temperatures

\begin{tabular}{cccccc}
\hline \multirow{2}{*}{$\mathrm{Mg}-10 \mathrm{wt} \% \mathrm{Bi}_{x} \mathrm{M}_{y} \mathrm{O}_{z}$} & \multicolumn{5}{c}{ 最大产氢速率 $\left(\mathrm{mL} \mathrm{g}^{-1} \mathrm{~min}^{-1}\right)$} \\
\cline { 2 - 6 } & $288.15 \mathrm{~K}$ & $298.15 \mathrm{~K}$ & $308.15 \mathrm{~K}$ & $318.15 \mathrm{~K}$ & $328.15 \mathrm{~K}$ \\
\hline $\mathrm{Bi}_{4} \mathrm{Ti}_{3} \mathrm{O}_{12}$ & 102.5 & 396.2 & 714.9 & 1362 & 2136.3 \\
$\mathrm{BiVO}_{4}$ & 61.4 & 300.7 & 360.2 & 962.5 & 1512.4 \\
$\mathrm{Bi}_{2} \mathrm{CrO}_{6}$ & 48.2 & 162.5 & 384.1 & 816.2 & 1320 \\
$\mathrm{Bi}_{2} \mathrm{MoO}_{6}$ & 362.2 & 564 & 993.2 & 2364.2 & 624.2 \\
$\mathrm{Bi}_{2} \mathrm{WO}_{6}$ & 48.5 & 112.1 & 276.2 & & 1272.5 \\
\hline
\end{tabular}

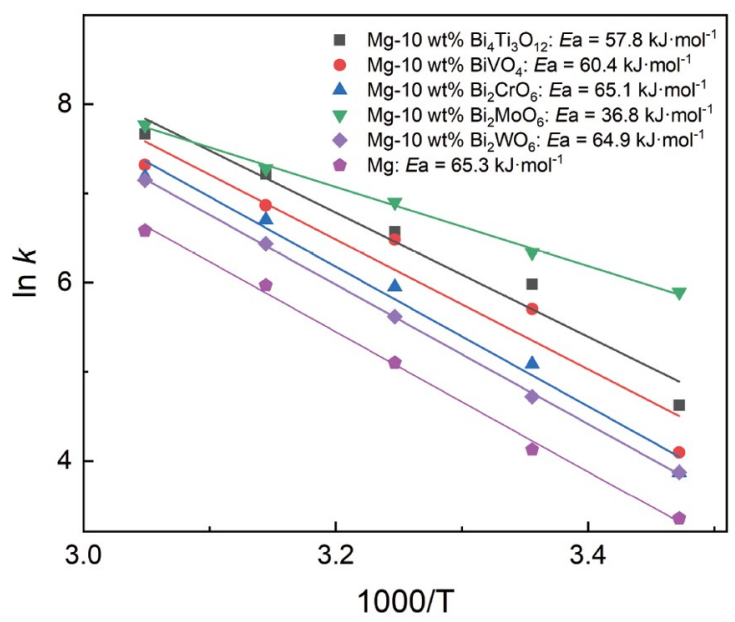

图 $3 \mathrm{Mg}-10 \quad \mathrm{wt} \% \quad \mathrm{Bi}_{x} \mathrm{M}_{y} \mathrm{O}_{z}$ 的 $\ln k$ 对 $1000 / T$ 拟合曲线(网络版 彩图)

Figure 3 The fitted curve of $\ln k v$ s. 1000/T for $\mathrm{Mg}-10 \mathrm{wt} \% \mathrm{Bi}_{x} \mathrm{M}_{y} \mathrm{O}_{z}$ (color online).

$298.15 \mathrm{~K}$ 下的产氢性能进行测试(图4). 从图4中可以 看出, $\mathrm{Bi}_{2} \mathrm{MoO}_{6}$ 含量为 $7 \mathrm{wt} \%$ 时具有最好的水解性能, 在 $100 \mathrm{~s}$ 内可生成 $555.2 \mathrm{~mL} \mathrm{~g}^{-1}$ 的氢气，而 $\mathrm{Mg}-x \mathrm{wt} \%$ $\mathrm{Bi}_{2} \mathrm{MoO}_{6}(x=3,5,10,13)$ 制氢材料在相同条件下可分 别生成298.1、358.4、474.1、443.1 $\mathrm{mL} \mathrm{g}^{-1}$ 的氢气. 反 应 $100 \mathrm{~s}$ 后, $\mathrm{Mg}-3 \mathrm{wt} \% \mathrm{Bi}_{2} \mathrm{MoO}_{6}$ 的氢气转化率为 $33.2 \%$, 进一步将 $\mathrm{Bi}_{2} \mathrm{MoO}_{6}$ 的含量提高到 $7 \mathrm{wt} \%$, 氢气转化率为 $63.9 \%$, 当再进一步增加 $\mathrm{Bi}_{2} \mathrm{MoO}_{6}$ 含量时, 氢气转化率 反而稍有下降. 显然, $\mathrm{Bi}_{2} \mathrm{MoO}_{6}$ 可以显著提高制氢材料 的初始产氢速率, 合理添加催化剂可以达到最佳水解 性能. 值得注意的是, $\mathrm{Mg}-7 \mathrm{wt} \% \mathrm{Bi}_{2} \mathrm{MoO}_{6}$ 制氢材料在 初始 $100 \mathrm{~s}$ 内的初始产氢性能和转化率均高于 $10 \mathrm{wt} \%$ 和13 $\mathrm{wt} \%$ 含量的制氢材料, 结果表明, 过多的 $\mathrm{Bi}_{2} \mathrm{MoO}_{6}$ 将覆盖制氢材料, 在一定程度上阻止了水解反应的连 续进行.

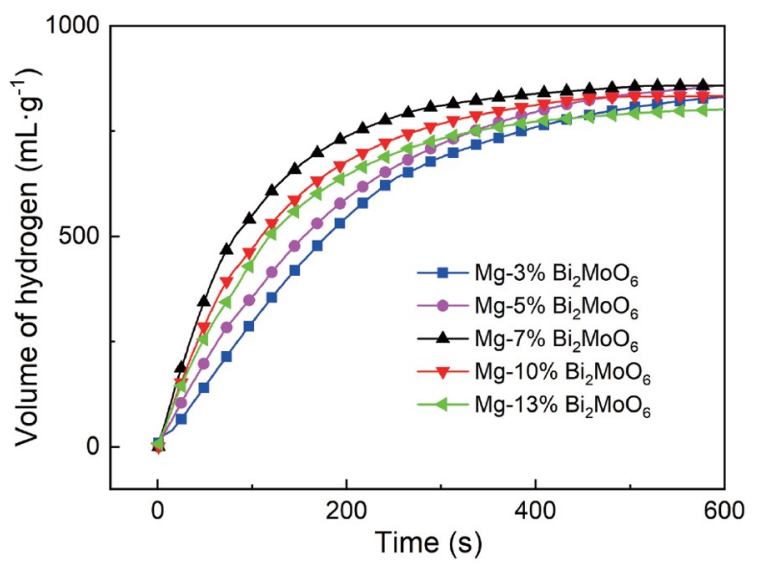

图 $4 \mathrm{Mg}-x \mathrm{wt} \% \mathrm{Bi}_{2} \mathrm{MoO}_{6}$ 的产氢曲线(网络版彩图)

Figure 4 The amount of hydrogen generation for $\mathrm{Mg}-x \mathrm{wt} \% \mathrm{Bi}_{2} \mathrm{MoO}_{6}$ (color online).

\section{$3.4 \mathrm{Bi}_{2} \mathrm{MoO}_{6} / \mathrm{CNTs}$ 的形貌及其复合 $\mathrm{Mg}$ 的水解产 氢性能分析}

为了进一步增加 $\mathrm{Mg}-\mathrm{Bi}_{2} \mathrm{MoO}_{6}$ 产氢性能, 通过水热 合成了 $\mathrm{Bi}_{2} \mathrm{MoO}_{6} / \mathrm{CNTs}$ 复合催化剂, 对其进行 $\mathrm{SEM}$ 与 TEM形貌分析, 微观结构如图5所示, 可以明显看出, $\mathrm{Bi}_{2} \mathrm{MoO}_{6}$ 负载在 $\mathrm{CNTs}$ 上, 证明成功制备了 $\mathrm{Bi}_{2} \mathrm{MoO}_{6} /$ CNTs. 从Mg-7 wt $\% \mathrm{Bi}_{2} \mathrm{MoO}_{6}$ 与 $\mathrm{Mg}-7 \mathrm{wt} \% \mathrm{Bi}_{2} \mathrm{MoO}_{6} /$ $\mathrm{CNTs}$ 在 $298.15 \mathrm{~K}$ 下的产氢曲线对比可以看出, 向 $\mathrm{Mg}$ $\mathrm{Bi}_{2} \mathrm{MoO}_{6}$ 体系引入 $\mathrm{CNTs}$ 可以有效增加 $\mathrm{Mg}$ 制氢材料的 初始产氢速率, 产氢曲线如图6所示, 在 $100 \mathrm{~s}$ 内氢气转 化率从 $63.9 \%$ 提高至 $85.5 \%$, 最大产氢速率从 756.1 提升 至 $2172.4 \mathrm{~mL} \mathrm{~g}^{-1} \mathrm{~min}^{-1}$.

\section{5 $\mathrm{Mg}-\mathrm{Bi}_{2} \mathrm{MoO}_{6} / \mathrm{CNTs}$ 复合材料的产氢动/热力学 分析}

进一步研究CNTs对 $\mathrm{Mg}-\mathrm{Bi}_{2} \mathrm{MoO}_{6}$ 水解反应活化能 


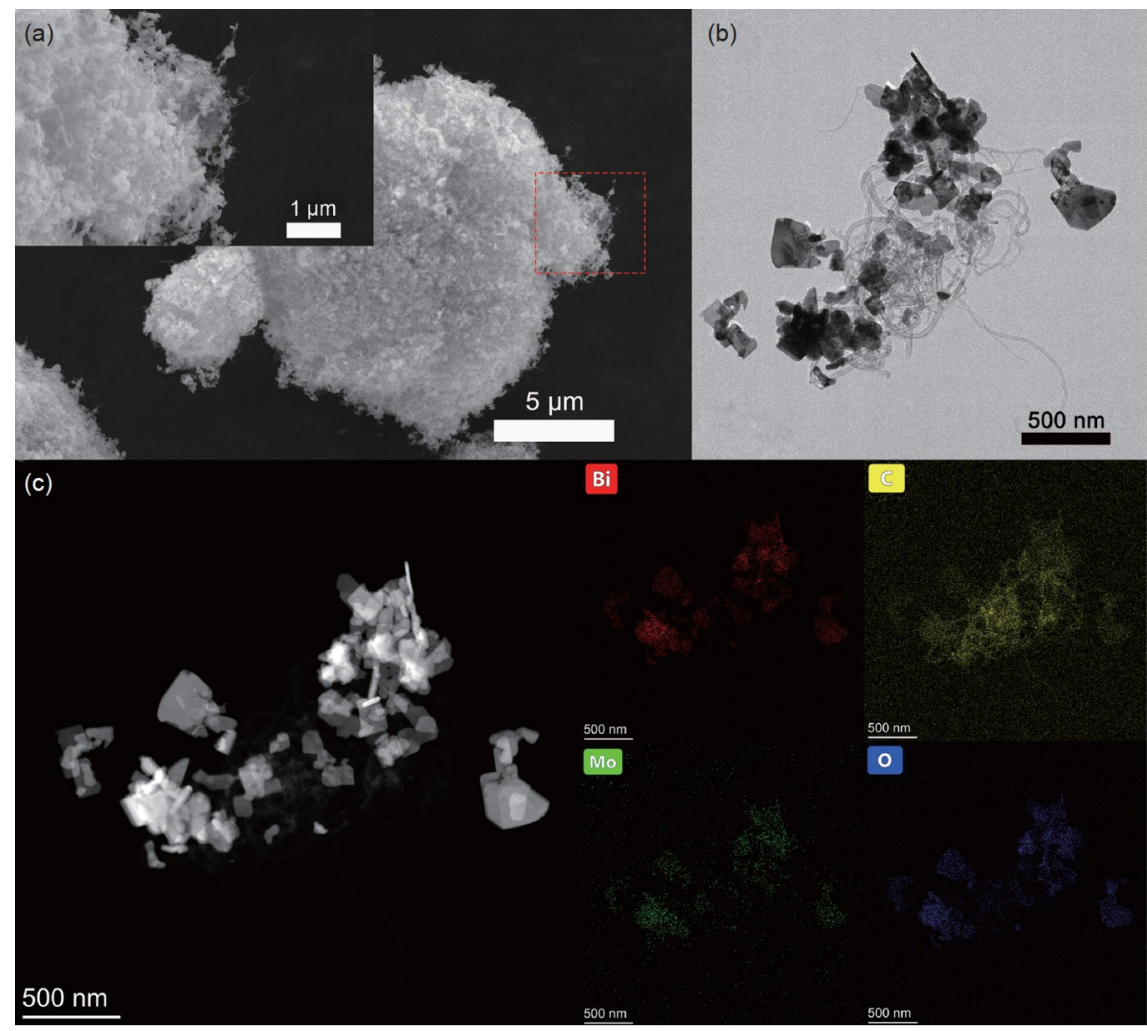

图 $5 \mathrm{Bi}_{2} \mathrm{MoO}_{6} / \mathrm{CNTs}$ 的SEM (a), TEM (b), EDS (c)图(网络版彩图)

Figure 5 (a) SEM, (b) TEM and (c) EDS images of $\mathrm{Bi}_{2} \mathrm{MoO}_{6} / \mathrm{CNTs}$ (color online).

的影响, 将球磨 $60 \mathrm{~min}$ 后的 $\mathrm{Mg}-7 \mathrm{wt} \% \mathrm{Bi}_{2} \mathrm{MoO}_{6}$ 与 $\mathrm{Mg}$ $7 \mathrm{wt} \% \mathrm{Bi}_{2} \mathrm{MoO}_{6} / \mathrm{CNTs}$ 分别在288.15、298.15、308.15、 318.15 和 $328.15 \mathrm{~K}$ 的 $3.5 \mathrm{wt} \% \mathrm{NaCl}$ 溶液进行水解反应. 根据阿伦尼乌斯公式，通过对两种制氢材料的反应速 率和对应的反应温度绘制 $1 n k-1000 / T$ 曲线，如图7所示, 通过向 $\mathrm{Mg}-\mathrm{Bi}_{2} \mathrm{MoO}_{6}$ 引入 CNTs, 活化能从34.9下降至 $23.6 \mathrm{~kJ} \mathrm{~mol}^{-1}$. 加入 CNTs可有效地增加制氢材料的反 应活性.

为了分析加入 $\mathrm{CNTs}$ 对 $\mathrm{Mg}-\mathrm{Bi}_{2} \mathrm{MoO}_{6}$ 制氢材料水解 反应过程产生的影响, 使用 $\mathrm{C} 80$ 量热仪测量了 $\mathrm{Mg}-7 \mathrm{wt} \%$ $\mathrm{Bi}_{2} \mathrm{MoO}_{6}$ 和 $\mathrm{Mg}-7 \mathrm{wt} \% \quad \mathrm{Bi}_{2} \mathrm{MoO}_{6} / \mathrm{CNTs}$ 在 $298.15 \mathrm{~K}$ 下水 解反应的反应热，结果如图8所示. 由图可知两种制氢 材料的反应热分别为 12.1 和 $12.4 \mathrm{~kJ} \mathrm{~g}^{-1}$ ，从两种材料的 热流曲线对比可知，引入CNTs后制氢材料的水解反应 更加剧烈, 初始水解反应速率增加, 根据文献 ${ }^{[32]}, \mathrm{Mg}$ 与 水反应的反应热约为 $14.2 \mathrm{~kJ} \mathrm{~g}^{-1}$, 结合测试数据可以得 出 Mg-7 wt $\% \mathrm{Bi}_{2} \mathrm{MoO}_{6} / \mathrm{CNTs}$ 的理论反应热应为 $13.1 \mathrm{~kJ} \mathrm{~g}^{-1}$. 可见, 两种材料的测量值低于其理论值, 这是由于样品在球磨过程中 $\mathrm{Mg}$ 与 $\mathrm{Bi}_{2} \mathrm{MoO}_{6}$ 发生固相

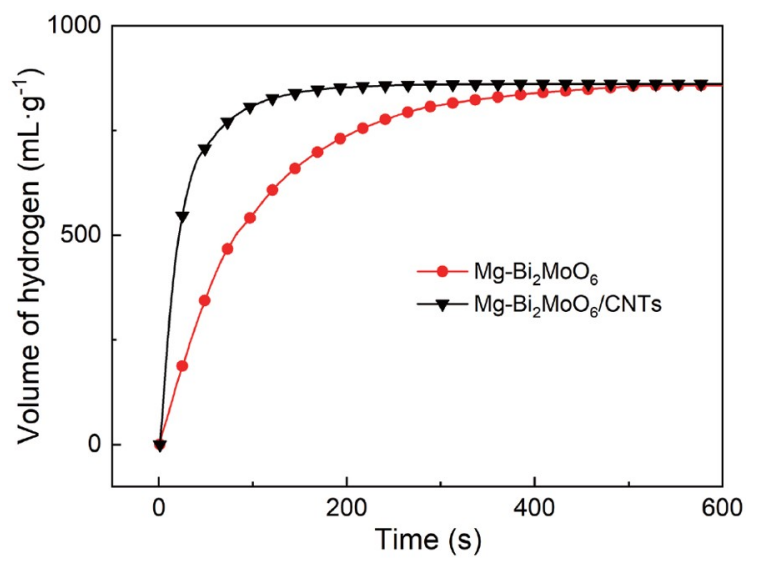

图 $6 \mathrm{Mg}-\mathrm{Bi}_{2} \mathrm{MoO}_{6}$ 与 $\mathrm{Mg}-\mathrm{Bi}_{2} \mathrm{MoO}_{6} / \mathrm{CNTs}$ 的产氢曲线(网络 版彩图)

Figure 6 Hydrogen generation curve of $\mathrm{Mg}-\mathrm{Bi}_{2} \mathrm{MoO}_{6}$ and $\mathrm{Mg}$ $\mathrm{Bi}_{2} \mathrm{MoO}_{6} / \mathrm{CNTs}$ (color online).

反应, 消耗了部分 $\mathrm{Mg}$.

\section{6 $\mathrm{Mg}-\mathrm{Bi}_{2} \mathrm{MoO}_{6} / \mathrm{CNTs}$ 的产氢机理分析}

为了进一步研究 $\mathrm{Bi}_{2} \mathrm{MoO}_{6} / \mathrm{CNTs}$ 对 $\mathrm{Mg}$ 水解产氢性 


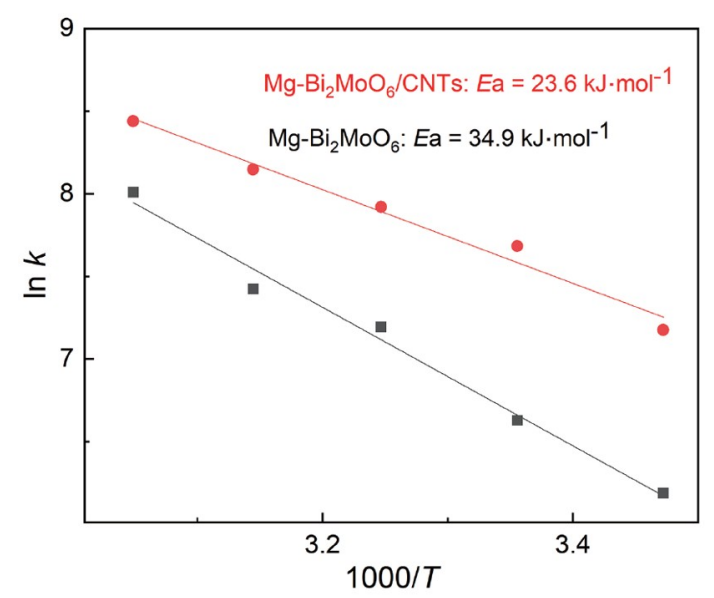

图 $7 \mathrm{Mg}-7 \mathrm{wt} \% \mathrm{Bi}_{2} \mathrm{MoO}_{6}$ 与 $\mathrm{Mg}-7 \mathrm{wt} \% \mathrm{Bi}_{2} \mathrm{MoO}_{6} / \mathrm{CNTs}$ 的 $\ln k$ 对 $1000 / T$ 拟合曲线(网络版彩图)

Figure 7 The curves of $\ln k v s .1000 / T$ for $\mathrm{Mg}-7 \mathrm{wt} \% \mathrm{Bi}_{2} \mathrm{MoO}_{6}$ and $\mathrm{Mg}-7 \mathrm{wt} \% \mathrm{Bi}_{2} \mathrm{MoO}_{6} / \mathrm{CNTs}$ (color online).

能的影响机制, 分别对不同球磨时间制备的 Mg-7 wt \% $\mathrm{Bi}_{2} \mathrm{MoO}_{6}$ 与 $\mathrm{Mg}-7 \mathrm{wt} \% \mathrm{Bi}_{2} \mathrm{MoO}_{6} / \mathrm{CNT}$ 进行 $\mathrm{SEM}$ 表面形 貌分析, 图9 a c 分别为球磨30、60和 $120 \mathrm{~min}$ 的 $\mathrm{Mg}$ $7 \mathrm{wt} \% \mathrm{Bi}_{2} \mathrm{MoO}_{6}$ 表面形貌，可以观察到随着球磨时间 的增加, $\mathrm{Mg}-7 \mathrm{wt} \% \mathrm{Bi}_{2} \mathrm{MoO}_{6}$ 尺寸有明显的增加, 逐渐 团聚形成金属薄片, 这是由于金属 $\mathrm{Mg}$ 具有很好的延展 性，当球磨时间增加到一定程度后再继续增加球磨时 间，会使 $\mathrm{Mg}$ 发生团聚现象，而 $\mathrm{Bi}_{2} \mathrm{MoO}_{6}$ 作为催化剂不 能有效地起到增加材料球磨性能的作用, 通过图 $9 \mathrm{~b}, \mathrm{~d}$ 的对比可以发现加入CNTs后球磨 $60 \mathrm{~min}$ 的 $\mathrm{Mg}$ 制氢材 料, 制氢材料的尺寸明显减小. 将 $\mathrm{Mg}-7 \mathrm{wt} \% \mathrm{Bi}_{2} \mathrm{MoO}_{6} /$ CNTs表面进行放大观察, 从图9e可以发现CNTs在球
磨后会覆盖在 $\mathrm{Mg}$ 粉末的表面上, 在球磨过程中可以起 到润滑保护作用，使 $\mathrm{Mg}$ 减少团聚，从而使制氢材料的 尺寸减小, 增加制氢材料与水的接触面积, 有利于水解 性能的提升.

使用XPS表征分析球磨60 min的 Mg-7 wt \% $\mathrm{Bi}_{2} \mathrm{MoO}_{6}$ 与 $\mathrm{Mg}-7 \mathrm{wt} \% \mathrm{Bi}_{2} \mathrm{MoO}_{6} / \mathrm{CNTs}$ 制氢材料表面 各个元素的化学价态. 图 $10 \mathrm{a}$ 为 $\mathrm{Mg}-7 \mathrm{wt} \% \mathrm{Bi}_{2} \mathrm{MoO}_{6}$ 的 $\mathrm{Mg} 2 \mathrm{p}$ 图谱, 其中只存在 $49.8 \mathrm{eV}$ 的 $\mathrm{Mg}$ 单质峰 ${ }^{[33]}$, 图 $10 \mathrm{~b}$ 为 $\mathrm{Mg}-7 \mathrm{wt} \% \mathrm{Bi}_{2} \mathrm{MoO}_{6} / \mathrm{CNTs}$ 的 $\mathrm{Mg} 2 \mathrm{p}$ 图谱, 分峰后发 现Mg-7 wt $\% \mathrm{Bi}_{2} \mathrm{MoO}_{6} / \mathrm{CNTs}$ 上存在着两种价态的 $\mathrm{Mg}$, 其中不仅存在 $49.8 \mathrm{eV}$ 的 $\mathrm{Mg}$ 单质峰, 还存在着属于 $\mathrm{MgO}$ 的 $50.9 \mathrm{eV}$ 的峰 ${ }^{[34]}$, 可以说明 $\mathrm{Mg}$ 粉末表面有部分 $\mathrm{Mg}$ 被氧化. 由图10c可以看出 $\mathrm{Mg}-7 \mathrm{wt} \% \mathrm{Bi}_{2} \mathrm{MoO}_{6}$ 在 $\mathrm{Bi} 4 \mathrm{f}$ 有两个分别为 159.3 和 $164.7 \mathrm{eV}$ 的峰, 属于 +3 价 $\mathrm{Bi}$ 的特征峰 ${ }^{[35]}$. 从图 $10 \mathrm{~d}$ 可以看出加入CNTs球磨后 $\mathrm{Bi} 4 \mathrm{f}$ 在 157.5 和 $162.7 \mathrm{eV}$ 处出现两个新的峰, 为 0 价单质 $\mathrm{Bi}$ 的特征峰 ${ }^{[36]}$, 图 $10 \mathrm{e}$ 为纯 $\mathrm{Bi}_{2} \mathrm{MoO}_{6}$ 的 +6 价的 $\mathrm{Mo}$ 特征 峰 ${ }^{[37]}$, 图10f为 $\mathrm{Mg}-7 \mathrm{wt} \% \mathrm{Bi}_{2} \mathrm{MoO}_{6} / \mathrm{CNTs}$, 对比可知, 两种制氢材料在球磨 $60 \mathrm{~min}$ 后 Mo元素没有出现新的 价态.

结合上述测试可以推断球磨时发生以下机理:

$3 \mathrm{Mg}+\mathrm{Bi}_{2} \mathrm{MoO}_{6} \rightarrow 3 \mathrm{MgO}+2 \mathrm{Bi}+\mathrm{MoO}_{3}$

$\mathrm{Mg}-7 \mathrm{wt} \% \mathrm{Bi}_{2} \mathrm{MoO}_{6} / \mathrm{CNTs}$ 制氢材料水解制氢的机 理可以解释如下. 首先, 通过水热法制备的 $\mathrm{Bi}_{2} \mathrm{MoO}_{6} /$ CNTs在球磨过程中CNTs起到了良好的助磨剂的作用, $\mathrm{CNTs}$ 覆盖在Mg粉末的表面阻止了 $\mathrm{Mg}$ 的团聚，促进了 水解反应进行. 其次在球磨中 $\mathrm{Mg}-7 \quad \mathrm{wt} \% \quad \mathrm{Bi}_{2} \mathrm{MoO}_{6} /$
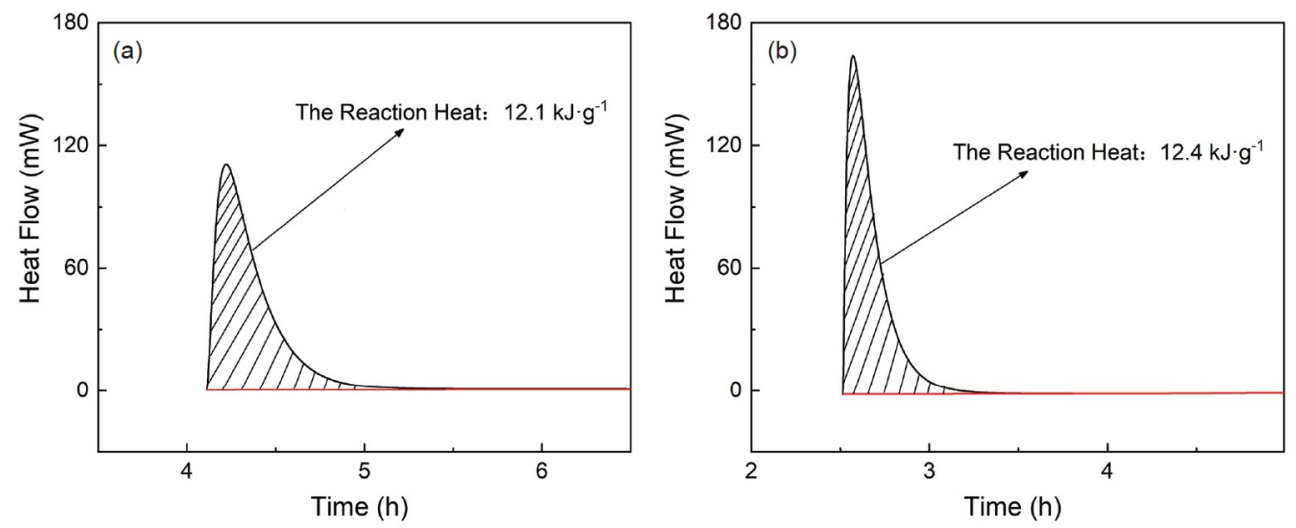

图 8 (a) Mg-7 wt $\% \mathrm{Bi}_{2} \mathrm{MoO}_{6}$ 和(b) Mg-7 wt $\% \mathrm{Bi}_{2} \mathrm{MoO}_{6} / \mathrm{CNTs}$ 水解的反应热(网络版彩图)

Figure 8 (a) Reaction heat of $\mathrm{Mg}-7 \mathrm{wt} \% \mathrm{Bi}_{2} \mathrm{MoO}_{6}$ and (b) $\mathrm{Mg}-7 \mathrm{wt} \% \mathrm{Bi}_{2} \mathrm{MoO}_{6} / \mathrm{CNTs}$ (color online). 

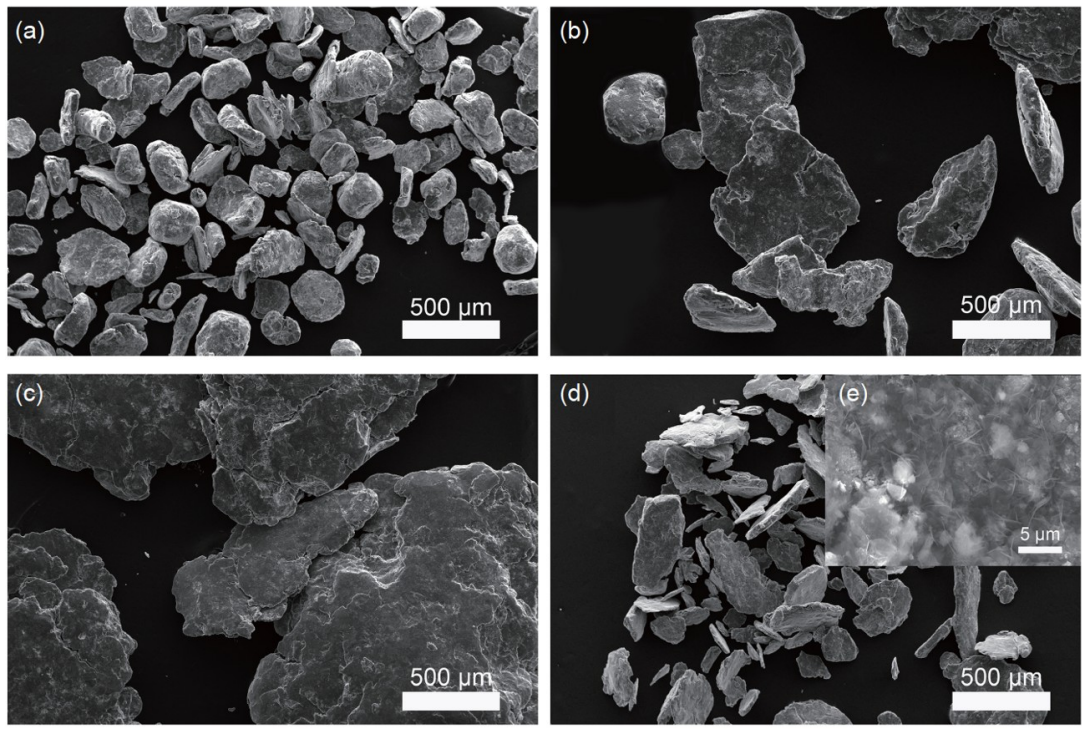

图 9 不同球磨时间制得的 $\mathrm{Mg}-7 \mathrm{wt} \% \mathrm{Bi}_{2} \mathrm{MoO}_{6}$ 与 $\mathrm{Mg}-7 \mathrm{wt} \% \mathrm{Bi}_{2} \mathrm{MoO}_{6} / \mathrm{CNTs}$ 的SEM图. (a) $30 \mathrm{~min}$; (b) $60 \mathrm{~min}$; (c) $120 \mathrm{~min}$; (d) CNTs 60 min; (e) CNTs 60 min表面

Figure 9 SEM images of $\mathrm{Mg}-7 \mathrm{wt} \% \mathrm{Bi}_{2} \mathrm{MoO}_{6}$ and $\mathrm{Mg}-7 \mathrm{wt} \% \mathrm{Bi}_{2} \mathrm{MoO}_{6} / \mathrm{CNTs}$ with different mill times. (a) $30 \mathrm{~min}$; (b) $60 \mathrm{~min}$; (c) $120 \mathrm{~min}$; (d) CNTs $60 \mathrm{~min}$; (e) Surface of the CNTs $60 \mathrm{~min}$.

$\mathrm{CNTs}$ 的 $\mathrm{Mg}$ 与 $\mathrm{Bi}_{2} \mathrm{MoO}_{6}$ 发生了固相反应，有部分 $\mathrm{Mg}$ 被 氧化成 $\mathrm{MgO}$ 并原位生成了 $\mathrm{MoO}_{3}$ 与单质 $\mathrm{Bi}$ ，产生的 $\mathrm{MoO}_{3}$ 同时也是 $\mathrm{Mg}$ 水解反应优良的催化剂 ${ }^{[29]}$, 其中高 活性单质 $\mathrm{Bi}$ 促进 $\mathrm{Mg}$ 催化水解反应不断进行, 而不加入 CNTs 只单纯地增长球磨时间会使 $\mathrm{Mg}$ 粉末团聚导致 $\mathrm{Mg}$ 与 $\mathrm{Bi}_{2} \mathrm{MoO}_{6}$ 发生固相反应的概率降低，上述所有因 素共同作用使得 $\mathrm{Mg}-7 \mathrm{wt} \% \mathrm{Bi}_{2} \mathrm{MoO}_{6} / \mathrm{CNTs}$ 具有良好的 水解制氢性能.

\subsection{DFT分析}

通过DFT计算研究 $\mathrm{Mg}$ 与 $\mathrm{Bi}_{2} \mathrm{MoO}_{6}$ 表面性质. 为了 修正材料总的偶极矩, 在 $Z$ 轴方向采用偶极矩修正. 为 了满足收敛条件，平面波基组扩展波函数截断能设置 为 $450 \mathrm{eV}$, 并且采用高斯展宽为 $0.05 \mathrm{eV}$. 在晶格和原子 弛豫过程中，计算的收玫判据是总能量差小于 $10^{-5} \mathrm{eV}$, 且各原子施加作用力小于 $0.02 \mathrm{eV} / \AA \AA$. 利用范德华尔斯 (DFT-D3)方法, 以修正材料表面分子间作用力. 为了避 免材料本身层与层间原子相互作用力影响, 在 $Z$ 轴方向 设置不少于 $15 \AA$ 的真空层. 使用 $2 \times 5 \times 1$ 的 $\mathrm{K}$ 格网点来计 算 $\mathrm{Bi}_{2} \mathrm{MoO}_{6}(010)$ 表面性质，采用 $3 \times 3 \times 1$ 的 $\mathrm{K}$ 格网点来进 行优化本征及 $\mathrm{Bi}$ 掺杂的 $\mathrm{Mg}(001)$ 晶面的基本性质, $5 \times 5 \times 1$ 的 $\mathrm{K}$ 格网点进行能量计算. 小分子 $\mathrm{H} 、 \mathrm{O} 、 \mathrm{OH}$ 和 $\mathrm{H}_{2} \mathrm{O}$ 的吸附能定义 $\left(E_{\mathrm{a}}\right)$ 如下:
$E_{\mathrm{a}}=E_{\text {total }}-E_{\text {molecular }}-E_{\text {substrate }}$

其中 $E_{\text {tota1 }}$ 代表小分子吸附在祄底表面时的系统总能 量, $E_{\text {molecular }}$ 表示吸附小分子 $\mathrm{H} 、 \mathrm{O} 、 \mathrm{OH}$ 和 $\mathrm{H}_{2} \mathrm{O}$ 的能量, $E_{\text {substrate }}$ 表示本征及 $\mathrm{Bi}$ 掺杂 $\mathrm{Mg}(001)$ 的能量.

根据计算的平均电位图(图11a，b), $\mathrm{Mg}(001)$ 显示 出比 $\mathrm{Bi}_{2} \mathrm{MoO}_{6}(010)$ 更大的功函数 $(\varphi)$ 和更低的费米能 级 $\left(E_{\mathrm{f}}\right)$, 表明 $\mathrm{Bi}_{2} \mathrm{MoO}_{6}(010)$ 的电子更容易逃逸. 当两种 材料相互接触时, $\mathrm{Bi}_{2} \mathrm{MoO}_{6}$ 的电子倾向于通过界面转 移到 $\mathrm{Mg}$ 表面，从而实现两种材料之间的费米能级平 衡. 此外, 我们进一步研究了 $\mathrm{H} 、 \mathrm{O} 、 \mathrm{OH}$ 和 $\mathrm{H}_{2} \mathrm{O}$ 小分子 在本征及 $\mathrm{Bi}$ 掺杂的 $\operatorname{Mg}(001)$ 面的吸附性能(表4). 计算 结果显示由于 $\mathrm{Bi}$ 原子的掺杂, $\mathrm{H}_{2} \mathrm{O}$ 在 $\mathrm{Mg}(001)$ 面吸附能 有一定的提升由 -0.53 提升至 $-0.55 \mathrm{eV}$, 同时, $\mathrm{H}$ 原子的 吸附能由-3.37降低至 $-0.12 \mathrm{eV}$. 如图 $11 \mathrm{c}$ 所示, 我们对 比了 $\mathrm{Bi}$ 原子掺杂前后 $\mathrm{Mg}(001)$ 面的态密度, 发现由于 $\mathrm{Bi}$

表 $4 \mathrm{H} 、 \mathrm{O} 、 \mathrm{OH}$ 和 $\mathrm{H}_{2} \mathrm{O}$ 在本征及 $\mathrm{Bi}$ 掺杂的 $\mathrm{Mg}(001)$ 面的吸 附能

Table 4 The absorption energy for $\mathrm{H}, \mathrm{O}, \mathrm{OH}$ and $\mathrm{H}_{2} \mathrm{O}$ on the pure and Bi-doped $\operatorname{Mg}(001)$ surface

\begin{tabular}{ccccc}
\hline Adsorption energies $(\mathrm{eV})$ & $\mathrm{H}$ & $\mathrm{O}$ & $\mathrm{OH}$ & $\mathrm{H}_{2} \mathrm{O}$ \\
\hline $\mathrm{Mg}(001)$ & -3.37 & -4.69 & -1.71 & -0.53 \\
Bi-doped $\mathrm{Mg}(001)$ & -0.12 & -5.09 & -1.80 & -0.55 \\
\hline
\end{tabular}



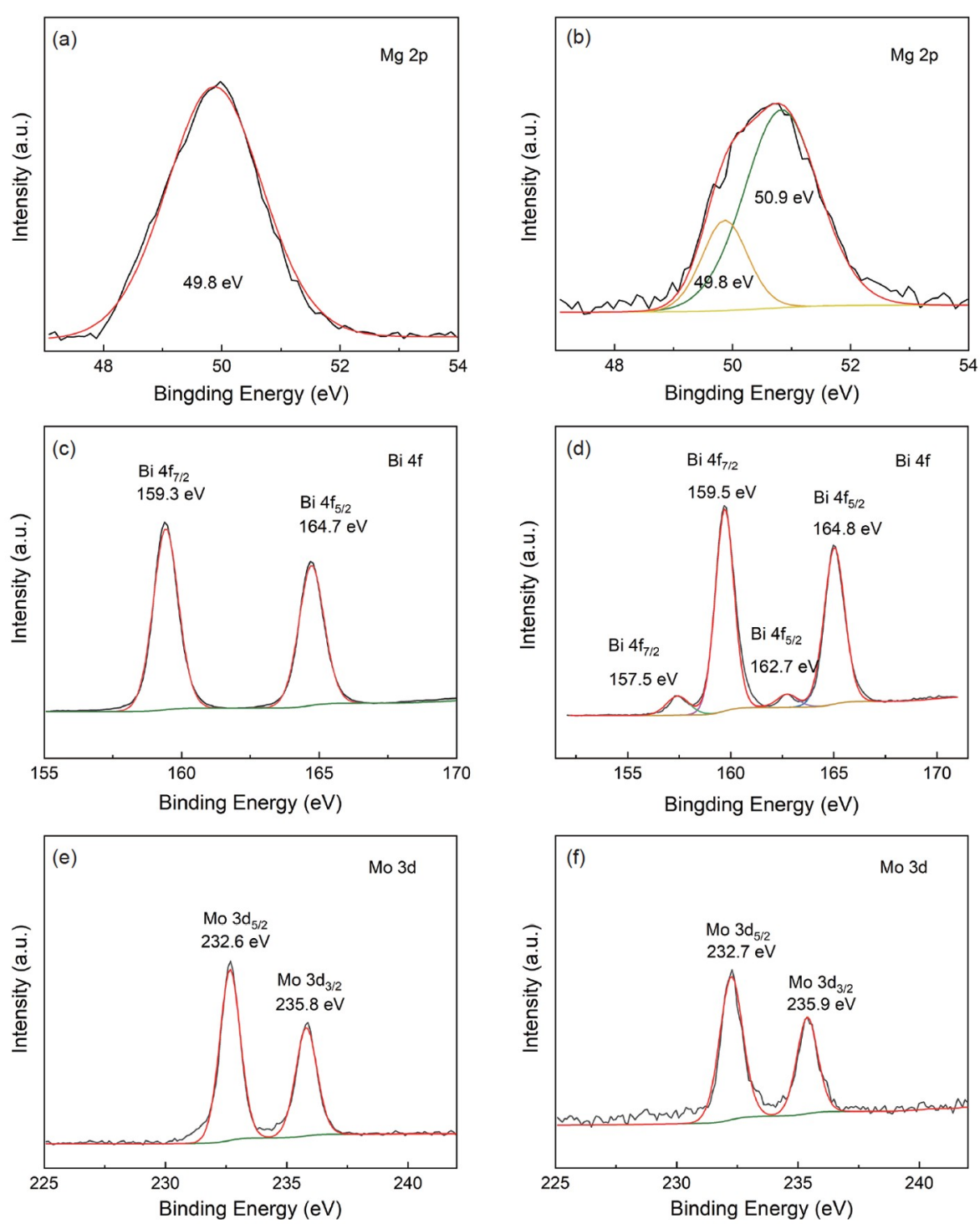

图 $10 \mathrm{Mg}-\mathrm{Bi}_{2} \mathrm{MoO}_{6}$ 与 $\mathrm{Mg}-\mathrm{Bi}_{2} \mathrm{MoO}_{6} / \mathrm{CNTs}$ 的XPS高分辨率谱图. (a) $\mathrm{Mg}_{-}-\mathrm{Bi}_{2} \mathrm{MoO}_{6} \mathrm{Mg} 2 \mathrm{p}$; (b) $\mathrm{Mg}-\mathrm{Bi}_{2} \mathrm{MoO}_{6} / \mathrm{CNTs} \mathrm{Mg} 2 \mathrm{p}$; (c) $\mathrm{Mg}$ $\mathrm{Bi}_{2} \mathrm{MoO}_{6} \mathrm{Bi} 4 \mathrm{f}$; (d) $\mathrm{Mg}-\mathrm{Bi}_{2} \mathrm{MoO}_{6} / \mathrm{CNTs} \mathrm{Bi} 4 \mathrm{f}$; (e) $\mathrm{Mg}-\mathrm{Bi}_{2} \mathrm{MoO}_{6}$ Mo 3d; (f) $\mathrm{Mg}-\mathrm{Bi}_{2} \mathrm{MoO}_{6} / \mathrm{CNTs}$ Mo $3 \mathrm{~d}$ (网络版彩图)

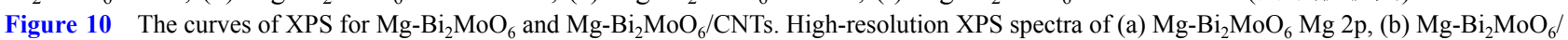
CNTs Mg 2p, (c) $\mathrm{Mg}-\mathrm{Bi}_{2} \mathrm{MoO}_{6} \mathrm{Bi} 4 \mathrm{f}$, (d) $\mathrm{Mg}-\mathrm{Bi}_{2} \mathrm{MoO}_{6} / \mathrm{CNTs} \mathrm{Bi} 4 \mathrm{f}$, (e) $\mathrm{Mg}_{-}-\mathrm{Bi}_{2} \mathrm{MoO}_{6} \mathrm{Mo} 3 \mathrm{~d}$, and (f) $\mathrm{Mg}_{-} \mathrm{Bi}_{2} \mathrm{MoO}_{6} / \mathrm{CNTs}^{\mathrm{Mo}} 3 \mathrm{~d}$ (color online).

原子的掺杂可提升 $\mathrm{Mg}$ 在费米能级的电荷分布, 有利于 $\mathrm{H}_{2} \mathrm{O}$ 的吸附. 上述研究表明, $\mathrm{Bi}$ 原子掺杂可改变 $\mathrm{Mg}$ 的 局域电荷分布, 可增强 $\mathrm{Mg}(001)$ 面对 $\mathrm{H}_{2} \mathrm{O}$ 的吸附能并降 低 $\mathrm{H}_{2} \mathrm{O}$ 解离后 $\mathrm{H}$ 原子的吸附能, 促进水解放氢反应的 发生.

\section{4 结论}

本文通过水热法制备了 $\mathrm{Bi}$ 系含氧酸盐 $\mathrm{Bi}_{x} \mathrm{M}_{y} \mathrm{O}_{z}(\mathrm{M}=$
Ti， V， Cr，Mo，W), 进而采用高能球磨法制备出 $\mathrm{Mg}-\mathrm{Bi}_{x} \mathrm{M}_{y} \mathrm{O}_{z}$ 复合制氢材料. 研究表明 $\mathrm{Mg}-\mathrm{Bi}_{2} \mathrm{MoO}_{6}$ 表现 出较好的产氢性能, 其最佳掺杂量为 $7 \mathrm{wt} \%$. 进一步研 究了 $\mathrm{Bi}_{2} \mathrm{MoO}_{6} / \mathrm{CNTs}$ 对 $\mathrm{Mg}$ 水解放氢性能的改善, 其放 氢活化能从 36.8 降低到 $23.6 \mathrm{~kJ} \mathrm{~mol}^{-1}$, 最大制氢速率从 756.1 提升至 $2172.4 \mathrm{~mL} \mathrm{~g}^{-1} \mathrm{~min}^{-1}$. 通过SEM表面形貌 分析可以得出增加球磨时间制氢材料发生明显的团 聚, 加入CNTs后复合制氢材料表面覆盖着CNTs且尺 寸明显减少. XPS分析表明 $\mathrm{Bi}_{2} \mathrm{MoO}_{6} / \mathrm{CNTs}$ 在与 $\mathrm{Mg}$ 的 

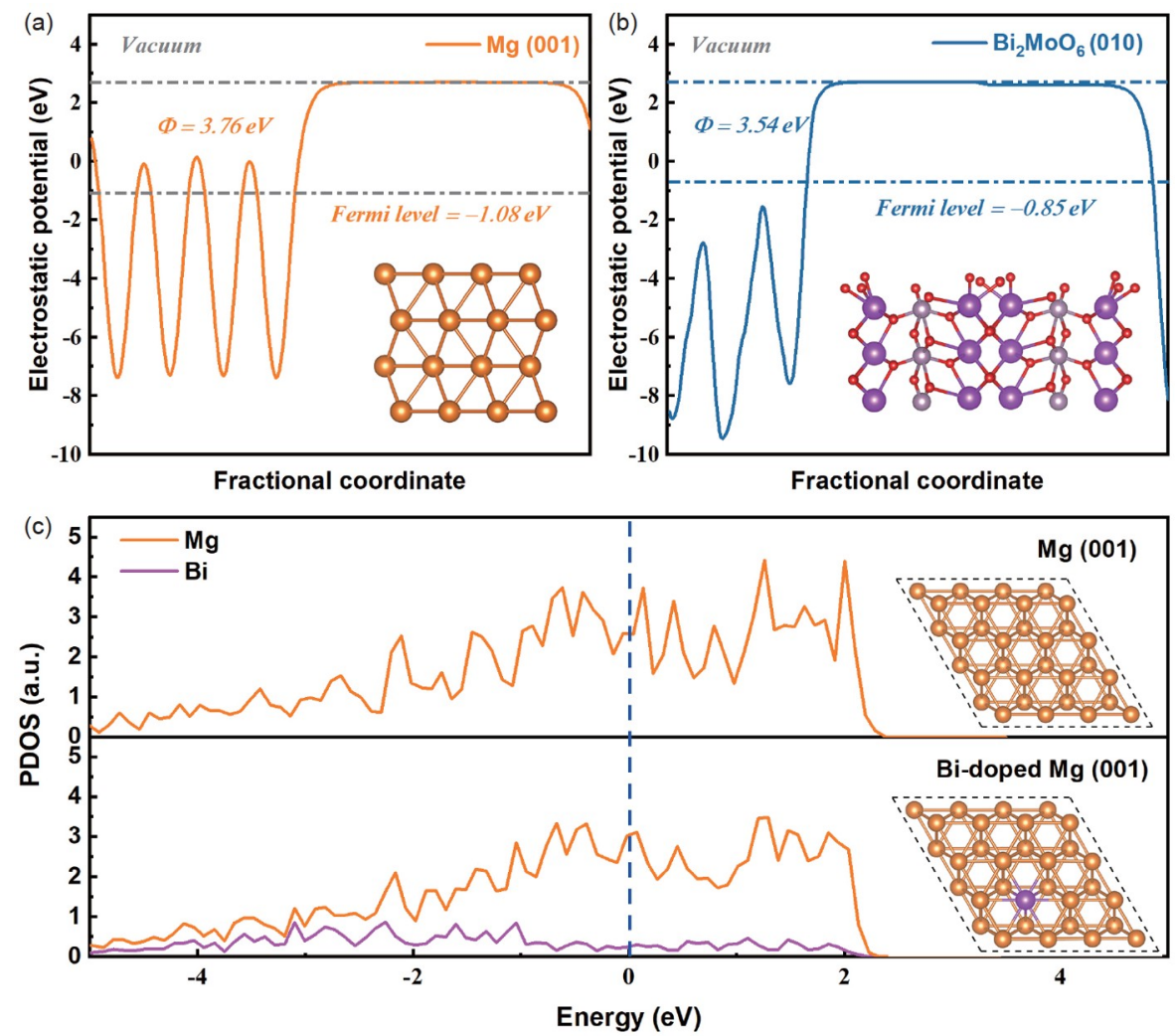

图 $11 \mathrm{Mg}(001)$ (a)和 $\mathrm{Bi}_{2} \mathrm{MoO}_{6}(010)$ (b)的平均电位图. (c) 本征和Bi掺杂的 $\mathrm{Mg}(001)$ 的分波态密度图(网络版彩图) Figure 11 Average potential profiles of (a) $\mathrm{Mg}(001)$ and (b) $\mathrm{Bi}_{2} \mathrm{MoO}_{6}(010)$. (c) Partial density of states for pure and $\mathrm{Bi}-\mathrm{doped} \mathrm{Mg}(001)$ (color online).

球磨过程中发生固相反应生成了Bi单质。根据DFT分 析计算得出Bi原子掺杂可改变 $\mathrm{Mg}$ 的局域电荷分布, 增
强 $\mathrm{Mg}$ 对 $\mathrm{H}_{2} \mathrm{O}$ 的吸附能并降低 $\mathrm{H}_{2} \mathrm{O}$ 解离后 $\mathrm{H}$ 原子的吸附 能, 促进水解反应的发生.

\section{参考文献}

1 Sun Y, Shen C, Lai Q, Liu W, Wang DW, Aguey-Zinsou KF. Energy Storage Mater, 2018, 10: 168-198

2 Durbin DJ, Malardier-Jugroot C. Int J Hydrogen Energy, 2013, 38: 14595-14617

3 Wang K, Pan Z, Yu X. J Alloys Compd, 2019, 794: 303-324

4 Zheng J, Yang DC, Li W, Fu H, Li X. Chem Commun, 2013, 49: 9437-9439

5 Abe JO, Popoola API, Ajenifuja E, Popoola OM. Int J Hydrogen Energy, 2019, 44: 15072-15086

6 Wang Z, Naterer GF. Int J Hydrogen Energy, 2014, 39: 14227-14233

7 Merki D, Fierro S, Vrubel H, Hu X. Chem Sci, 2011, 2: 1262-1267

8 Kannan MB. Surf Coatings Tech, 2016, 301: 36-41

9 Giang TT, Lunprom S, Liao Q, Reungsang A, Salakkam A. PeerJ, 2019, 7: e6637

10 Xie X, Li Y, Chen M, Hu M, Feng Y, Shang J, Liu T. ACS Sustain Chem Eng, 2020, 8: 4920-4930

11 Shen C, Aguey-Zinsou KF. J Mater Chem A, 2017, 5: 8644-8652

12 Yilmaz C, Kanoglu M, Abusoglu A. Int J Hydrogen Energy, 2015, 40: 14021-14030

13 Kushch SD, Kuyunko NS, Nazarov RS, Tarasov BP. Int J Hydrogen Energy, 2011, 36: 1321-1325

14 Meroueh L, Eagar TW, Hart DP. ACS Appl Energy Mater, 2020, 3: 1860-1868 
Al Bacha S, Awad AS, El Asmar E, Tayeh T, Bobet JL, Nakhl M, Zakhour M. Int J Hydrogen Energy, 2019, 44: 17515-17524

Ouyang L, Ma M, Huang M, Duan R, Wang H, Sun L, Zhu M. Energies, 2015, 8: 4237-4252

Awad AS, El-Asmar E, Tayeh T, Mauvy F, Nakhl M, Zakhour M, Bobet JL. Energy, 2016, 95: 175-186

Huang M, Ouyang L, Chen Z, Peng C, Zhu X, Zhu M. Int J Hydrogen Energy, 2017, 42: 22305-22311

He T, Chen W, Wang W, Du S, Deng S. J Alloys Compd, 2020, 827

Ouyang LZ, Huang JM, Wang H, Wen YJ, Zhang QA, Sun DL, Zhu M. Int J Hydrogen Energy, 2013, 38: 2973-2978

Oh SK, Kim MJ, Eom KS, Kyung JS, Kim DH, Cho EA, Kwon HS. Int J Hydrogen Energy, 2016, 41: 5296-5303

Hiraki T, Hiroi S, Akashi T, Okinaka N, Akiyama T. Int J Hydrogen Energy, 2012, 37: 12114-12119

Grosjean M, Zidoune M, Huot J, Roue L. Int J Hydrogen Energy, 2006, 31: 1159-1163

Kantürk AF, Coşkuner B, Pişkin S. Int J Hydrogen Energy, 2015, 40: 7483-7489

Liu Y, Wang X, Liu H, Dong Z, Cao G, Yan M. Energy, 2014, 68: 548-554

Liu Y, Wang X, Dong Z, Liu H, Li S, Ge H, Yan M. Energy, 2013, 53: 147-152

Sun Q, Zou M, Guo X, Yang R, Huang H, Huang P, He X. Energy, 2015, 79: 310-314

Wang S, Sun LX, Xu F, Jiao CL, Zhang J, Zhou HY, Huang FL. Int J Hydrogen Energy, 2012, 37: 6771-6775

Huang M, Ouyang L, Ye J, Liu J, Yao X, Wang H, Shao H, Zhu M. J Mater Chem A, 2017, 5: 8566-8575

Ma M, Yang L, Ouyang LZ, Shao H, Zhu M. Energy, 2019, 167: 1205-1211

Wang T, Xu F, Sun LX, Miao L, Liao L, Wei S, et al. J Alloys Compd, 2021, 860

Xu S, Liu J. Front Energy, 2018, 13: 27-53

Fournier V, Marcus P, Olefjord I. Surf Interface Anal, 2002, 34: 494-497

Yao HB, Li Y, Wee ATS, Pan JS, Chai JW. Appl Surf Sci, 2001, 173: 54-61

Li J, Yin Y, Liu E, Ma Y, Wan J, Fan J, Hu X. J Hazard Mater, 2017, 321: 183-192

Ding J, Wang H, Luo Y, Xu Y, Liu J, Lin Y. Catalysts, 2020, 10: 389

Kandi D, Behera A, Sahoo S, Parida K. Sep Purif Tech, 2020, 253: 117523 


\title{
Kinetic/thermodynamic study on improved hydrolysis hydrogen generation of Mg-doped bismuth series oxysalt
}

\author{
Jie Lin $^{\dagger}$, Jiaxi Liu ${ }^{\dagger}$, Lixian Sun ${ }^{*}$, Fen Xu', Yumei Luo, Yongpeng Xia, Chenchen Zhang, \\ Riguang Cheng, Sheng Wei, Pengru Huang, Bin Li, Kexiang Zhang, Dan Cai \\ Guangxi Key Laboratory of Information Materials \& Guangxi Collaborative Innovation Centre of Structure and Property for New Energy and \\ Materials, School of Material Science and Engineering, Guilin University of Electronic Technology, Guilin 541004, China \\ $\dagger$ These authors contributed equally to this work. \\ *Corresponding authors (email: sunlx@guet.edu.cn; xufen@guet.edu.cn)
}

\begin{abstract}
Study of high efficiency hydrogen production materials has attracted many researchers' attention. Mg-based hydrogen production material has the advantages of wide sources, mild reaction, simple process, safety control, and high hydrogen production capacity. In this article, $\mathrm{Mg}$ was doped with bismuth series oxysalt $\mathrm{Bi}_{x} \mathrm{M}_{y} \mathrm{O}_{z}(\mathrm{M}=\mathrm{Ti}, \mathrm{V}, \mathrm{Cr}, \mathrm{Mo}, \mathrm{W})$ by high energy ball milling method to improve the performance of hydrolytic hydrogen generation for the first time. It was found that $\mathrm{Bi}_{2} \mathrm{MoO}_{6} / \mathrm{CNTs}$ enhanced the hydrogen generation performance of $\mathrm{Mg}$ and increased the initial hydrogen generation rate. The optimal hydrogen generation rate increased to $2172.4 \mathrm{~mL} \mathrm{~g}^{-1} \mathrm{~min}^{-1}$, and the related activation energy decreased to $23.6 \mathrm{~kJ} \mathrm{~mol}^{-1}$. Density functional theory (DFT) calculation found that doped Bi atom enhanced the adsorption of $\mathrm{H}_{2} \mathrm{O}$ on $\mathrm{Mg}$ and reduced the adsorption of $\mathrm{H}$ atom by changing the local charge distribution, thus promoting the hydrolysis reaction.
\end{abstract}

Keywords: hydrolytic hydrogen generation, $\mathrm{Mg}$, bismuth series oxysalt, kinetics, thermodynamics

doi: $10.1360 /$ SSC-2021-0251 